\title{
“SPOTTEANDO" PALABRAS: LA TRANSFERENCIA SPOTTER EN LA OBSERVACIÓN Y FOTOGRAFÍA DE AERONAVES Y EL AUTOMOVILISMO
}

\section{"Spotting" words: spotter in aircraft observation and photography and in motor racing}

\author{
Lorena M.A. De- Matteis* \\ Universidad Nacional del Sur - CONICET \\ 1matteis@uns.edu.ar
}

Palabras clave

español;

transferencia léxica;

anglicismo;

aviación;

automovilismo

Keywords

Spanish;

lexical borrowing;

anglicism;

aviation;

motor racing

\section{Resumen}

En el marco de estudios léxicos que buscan iluminar aspectos de las representaciones sociales sobre el transporte aéreo, este trabajo pretende contribuir al conocimiento del léxico de origen foráneo empleado por hablantes de español. Propone una aproximación al uso de la transferencia léxica de origen inglés spotter en relación con dos actividades: la observación y registro fotográfico de aeronaves y el automovilismo deportivo. A partir del análisis de su empleo y en textos periodísticos y producciones de hablantes de español en la red social Twitter, se concluye que spotter es una voz que no se encuentra extendida en el uso general de la lengua pero que sí permite la identificación de grupo de quienes practican estas actividades.

\begin{abstract}
Within the framework of lexical studies that seek to illuminate aspects of social representations about air transport, this work aims to increase knowledge about foreign words employed by Spanish speakers. It proposes an approach to the use of spotter in relation with two activities: observation and photographic record of aircraft, and motor racing. Analysis of spotter in journalistic texts and productions of Spanish speakers on the social network Twitter, shows that this lexical unit has not yet become widespread in the general use of language, although it does signal group identity among those speakers who participate of these activities.
\end{abstract}




\section{"Spotteando" palabras: la transferencia spotter en la observación y fotografía de aeronaves y el automovilismo}

\section{Introducción}

Para el estudio de las representaciones sociales en torno al transporte aéreo, el estudio del léxico se constituye en una herramienta fundamental puesto que las denominaciones de los vehículos, las personas y las acciones vinculadas con el vuelo ilustran tanto la recepción de las novedades tecnológicas, como las valoraciones sociales que reciben esta modalidad del transporte y sus profesionales (cfr. por ej. de- Matteis, 2014, 2016). Al mismo tiempo, el léxico pone en evidencia las influencias técnicas y lingüísticas que se producen en el terreno aeronáutico (cfr. de- Matteis, 2018, 2019a, 2019b).

En dicho marco, este trabajo pretende también contribuir al conocimiento del léxico de origen foráneo empleado por hablantes de español y para ello se propone, en primer lugar, explorar el empleo de la transferencia spotter en relación con un pasatiempo vinculado con ámbito aeronáutico: la observación y registro fotográfico de aeronaves. Si bien nuestro trabajo se centra en el contexto aeronáutico, el rastreo de la expresión spotter en la red social Twitter -véase infra-alertó sobre la conveniencia de contrastar su empleo también en el campo del deporte motor, contexto de uso que se explora en segundo lugar y más brevemente en este trabajo.

Con relación a estos dos contextos de uso, ambos vinculados con actividades recreativas asociadas con estas modalidades del transporte, cabe realizar algunas observaciones preliminares que justifican su tratamiento conjunto. En primer lugar, el automovilismo es un deporte que presenta al menos dos importantes aspectos en común con la aviación: la relevancia del continuo desarrollo técnico y el consecuente profesionalismo de las grandes automotrices, por un lado, y la figura del "piloto", como exponente visible (y reconocible, en este caso) de una actividad que requiere la labor conjunta de equipos de trabajo numerosos y diversos. En el plano lingüístico, por otra parte, los hablantes manejan un vocabulario que, por su origen técnico y según el grado de formación e implicación de los hablantes con los mundos de la aviación y del automovilismo, puede caracterizarse como de tipo jergal o incluso profesional. Además, tanto el terreno del transporte como el del deporte -que se intersecan en el caso del automovilismo deportivo- se caracterizan por incorporar con frecuencia voces del inglés (Fontanella de Weinberg, 1992), ${ }^{1}$ que pueden recibir distinto grado de adaptación estructural a las pautas fonológicas y morfosintácticas del español. El caso de spotter, y de algunas formas léxicas relacionadas que se mencionarán en el análisis que sigue, resulta claramente ilustrativo de este fenómeno.

1. Es de interés observar que Fontanella de Weinberg señala como campos léxicos especialmente receptivos para los préstamos del inglés durante el siglo XX tanto al automovilismo como a la aviación, como así también a los deportes (1992, p. 174). 


\section{Consideraciones metodológicas}

En un marco general de estudios sobre préstamos léxicos, y tomando como base la distinción entre transferencias entre sistemas lingüísticos sin integración social (interferencias) e integradas al sistema de la lengua receptora (préstamos) ${ }^{2}$, en nuestros trabajos tenemos como premisa que la prensa tiene un rol preponderante en la difusión de voces de carácter terminológico-características de las lenguas de especialidad- en la lengua general (Gómez de Enterría, 2009, p. 22-23). Por este motivo, los ejemplos de uso de spotter proceden, en primer lugar, de textos periodísticos de diversas variedades de español que integran un corpus especializado diseñado como insumo para construir una base de datos léxicos destinada a la elaboración de un Léxico español del vuelo (de- Matteis, 2017). La mayor parte de los textos procesados -más de mil- corresponden al período 2007-2017 y fueron recuperados, sobre todo, mediante las plataformas en línea de diversos diarios de países de habla hispana. ${ }^{3}$

A partir de los datos obtenidos en este corpus, en el que detectamos las unidades léxicas spotter y spotting, se propusieron otras formas hipotéticas relacionadas. En otras palabras, se configuró una lista de posibilidades de representación gráfica y de derivación de spotter, con distinto grado de adaptación a las pautas morfogramaticales del español para corroborar su empleo entre hablantes de español. En esta lista, además de variantes gráficas de spotter, se consideró la existencia de un sustantivo deverbal -spotteo- y un verbo -spottear-, para los que también se propusieron diversas representaciones escritas (véase Tabla 1$){ }^{4}$

El empleo de todas estas formas se rastreó, en primer lugar, en el Corpus de Referencia del Español Actual (CREA) y el Corpus del Español del siglo XXI en línea (CORPES XXI), y, en segundo lugar, en la red social Twitter. Consideramos que, como herramientas de investigación, los mencionados corpus digitales permiten superar el sesgo disciplinar de nuestro corpus especializado de prensa aeronáutica y alertan sobre otros posibles usos de las unidades léxicas detectadas en los textos periodísticos. Si bien estos instrumentos ofrecen información cuantitativa, en este artículo se prioriza la perspectiva cualitativa de análisis.

2. Cfr. en este punto los aportes de Hipperdinger (2001, p. 14).

3. Las variedades más representadas en este corpus son las de Argentina -con predominio de la bonaerense- y de España -con predominio de la castellana-. Además, integran este corpus textos periodísticos de Bolivia, Colombia, Chile, Cuba, Ecuador, México, Perú, Uruguay y Venezuela.

4. Si bien no trabajamos con datos de la oralidad, en su formulación se trabajó también con formas que respetan las pautas del español para el uso de la tilde en las voces graves y otras que no. 


\begin{tabular}{|l|l|l|}
\hline Forma léxica & \multicolumn{1}{|c|}{ Significado } & \multicolumn{1}{c|}{ Formas hipotéticas } \\
\hline spotter & $\begin{array}{l}\text { Denominación de } \\
\text { persona. }\end{array}$ & "spóter/spoter/espótter/espotter/espóter/espoter \\
\hline spotting & $\begin{array}{l}\text { Denominación de } \\
\text { actividad. }\end{array}$ & "spóting/spotting/espótting/espotting/espóting/espoting \\
\hline${ }^{*}$ spotteo & $\begin{array}{l}\text { Denominación de } \\
\text { actividad. }\end{array}$ & "spotteo/spoteo/espotteo/espoteo \\
\hline${ }^{*}$ spottear & Acción. & "spottear/spotear/espottear/espotear \\
\hline
\end{tabular}

Tabla 1. Formas hipotéticas planteadas ${ }^{5}$

Por su parte, la brevedad de las contribuciones que admite la red social Twitter, potencia -al menos en principio- la selección de las voces que los hablantes consideran como las más adecuadas para su intención comunicativa. Así, apoyándonos en el ejemplo de investigaciones previas que han demostrado la utilidad de los textos publicados en esta red para estudios léxicos (Moreno Fernández y Moreno Sandoval, 2017), consideramos que esta red social permite, estudiar los usos de spotter, spotting y de las formas hipotéticas formuladas como variantes gráficas o como derivados léxicos en producciones no periodísticas de hablantes de español. ${ }^{6}$

\section{Análisis}

\subsection{La voz spotter: caracterización general de su empleo en español}

Derivada del verbo to spot (siglo XII-XIII), una de las acepciones del Webster's Encyclopedic Unabridged Dictionary of the English Language para spotter (spot +-er, siglo XVII) es la que corresponde al 'soldado que identifica los objetivos para los francotiradores'. ' Los diccionarios de inglés consignan también una acepción propia de la defensa civil, para la que este nombre designa a la 'persona que vigila la aparición de aeronaves enemigas'. En ambos casos, las acepciones de spotter comparten la idea central de 'avistar' u 'observar' algo difícil de detectar o que se está buscando de manera intencional. Estas ideas se transfieren a los ámbitos de uso que se identificaron para este trabajo, tanto el de la observación y fotografía de aeronaves como el del automovilismo.

Como designación de persona, y en lo que hace a la adecuación morfogramatical de este lexema, hemos registrado el empleo de spotter tanto con género masculino como femenino (por ejemplo, en Twitter "un spotter", "mi spotter + adjetivo de género femenino"). En el plural hemos registrado la forma spotters, aunque se ha constatado también alguna construcción en la que no hay

5. Las voces hipotéticas se indican con * en esta tabla, con independencia de que luego se haya constatado su uso o no, para distinguirlas de las observadas inicialmente en el corpus periodístico.

6. Los datos de esta red social se presentarán en forma agregada, esto es, bajo la forma de sintagmas aislados de su contexto, para evitar la trazabilidad de los mensajes y su atribución a usuarios individuales.

7. Las traducciones de estas acepciones son propias. 
concordancia de número, lo que puede sugerir la duda del hablante con respecto a la forma de plural ("nuestros colegas spotter").

Para finalizar, el verbo inglés tiene como posibles equivalentes semánticos en español a los verbos observary avistar. Sin embargo, si bien hemos constatado en el uso expresiones como "avistaje de aviones", no constatamos nunca el empleo de *avistador y solo algunas veces el de observador en los contextos a los que nos referimos en este artículo. Por otra parte, puede argumentarse que estas expresiones españolas no evocan de manera directa la fotografía de los objetos avistados, matiz de sentido siempre presente en el uso de spotter en el caso de la observación de aeronaves, ámbito en el que los avistajes se realizan con el fin último y característico del registro visual de distintos modelos de aeronaves y, muchas veces, de su esquema de pintura o livery (de- Matteis, 2018).

\subsection{Observaciones cuantitativas}

Aunque el análisis que se presenta en las siguientes secciones en esencia cualitativo, ofrecemos algunos datos como referencia sobre la frecuencia de uso de las transferencias detectadas en el corpus de prensa aeronáutico (Tabla 2).

Como es lógico esperar, por su carácter especializado, este corpus ofrece más casos de spotter que los corpus digitales. En ellos, los casos registrados se corresponden, en su gran mayoría, con el significado militar del anglicismo. En tal sentido, la cita del texto de ficción que en más casos presenta esta voz es muy ilustrativa y ofrece en sintagmas sucesivos el empleo de la forma foránea y de su posible equivalente semántico en español dentro del terreno militar, esto es, 'avistador':

El sniper y el spotter. El tirador y el avistador. El segundo pone el ojo y el primero pone la bala. E1 primero aprieta el gatillo recién después que el segundo le ha proporcionado las circunstancias perfectas para dar en el blanco: condiciones atmosféricas, distancia del objetivo, posibles variables de último momento. (Fresan, 2009, el destacado nos pertenece)

\begin{tabular}{|l|l|l|l|}
\hline Transferencia & $\begin{array}{l}\text { Corpus de prensa } \\
\text { aeronáutica }\end{array}$ & CREA & CORPES XXI $^{8}$ \\
\hline spotter(s) & $28 / 7$ & $5 / 1^{9}$ & $8 / 2^{10}$ \\
\hline spotting & $6 / 3$ & sin registros & sin registros relevantes \\
\hline
\end{tabular}

Tabla 2. Frecuencia de uso de formas léxicas básicas

8. Se consultó la versión 0.92 de este corpus, correspondiente a mayo de 2020.

9. Todos los ejemplos, de la novela Madera de héroe de Miguel Delibes, designan a un instrumento militar para la observación. 10. Una de las 5 formas de singular corresponde a un artículo de investigación sobre anglicismos en España (Seco 20002001), mientras que todas las restantes, incluyendo tres casos del plural spotters, corresponden al terreno militar, como en el ejemplo ofrecido en el Diccionario del Español Actual citado.

11. La única ocurrencia se corresponde con el uso en ginecología de este anglicismo, como 'el manchado o sangrado entre menstruaciones'. 
Pese a la baja frecuencia de uso de spotter en ambos corpus, el Diccionario del Español Actualincluye un artículo lexicográfico para esta forma léxica, aunque la definición resulte algo vaga por recurrir al adjetivo "cierto" para modificar con sentido de indeterminación a la expresión nominal que califica:

spotter (ing; pronunc corriente, /espóter/; pl normal, s) $m$ (Mil) Cierto aparato de observación. | Delibes Madera 351: Fondeados en la bahía, durante las guardias, pensaba en ella mientras repasaba con el spotter el perfil de la ciudad.

En este diccionario, entonces, la voz no designa a una persona sino a un instrumento utilizado para la observación en el ámbito militar. Ni spotter ni spotting aparecen, por otro lado, en el Diccionario de la Lengua Española, ni en ninguna de las herramientas lexicográficas ofrecidas por el Nuevo Tesoro Lexicográfico de la Lengua Española. ${ }^{12}$

\subsubsection{Formas hipotéticas}

En lo que hace a las variantes léxicas y gráficas hipotetizadas para su exploración, solamente espotear presenta una ocurrencia en cada corpus digital. En ningún caso se vincula su empleo con la actividad de fotografía aérea ni con el automovilismo deportivo. En el CREA, el ejemplo se encuentra en una reflexión metalingüística acerca de nuevos verbos registrados en el español realizada en un texto periodístico de Bolivia de 1997. Tanto en este ejemplo como en el detectado en el CORPES XXI, correspondiente a una novela publicada en el año 2011 en México, la forma adaptada del verbo tiene un sentido que se aproxima a la idea 'observar', 'avistar' o 'ubicar en un grupo'. ${ }^{13}$ Con este significado, la expresión de préstamo parece tener integración social en algunas variedades del español, en particular en la mexicana.

Además, la búsqueda de la forma hipotética *spóting/spoting, como posibles variantes gráficas de spotting, da por resultado una única ocurrencia en CORPES XXI -en realidad, ambas búsquedas se corresponden con el mismo resultado. Sin embargo, este resultado representa un falso positivo pues se trata de un error de tipeo por la voz sporting.

Ahora bien, si la mayoría de las formas hipotéticas postuladas no se registran en los corpus digitales ${ }^{14}$ en los apartados que siguen se realizarán también observaciones sobre el empleo de las que

12. De manera complementaria, una consulta realizada en mayo de 2021 a través de la plataforma en línea del observatorio neológico correspondiente al buscador Obneo (desarrollado por la Universitat Pompeu Fabra) arroja cuatro ocurrencias diferentes para spotter en prensa escrita desde 1998. Las tres últimas -fechadas entre 2014 y 2020 - se corresponden con el contexto de la fotografía aeronáutica. La ocurrencia de 1998, en cambio, corresponde a un texto periodístico en el que se alude a una situación de seguridad pública, empleo que no tenemos registrado en nuestros datos pero que puede vincularse con la acepción militar de la voz en lengua inglesa: "Estética aparte, el día fue tranquilo. Sólo hay constancia de tres detenciones entre los ingleses, reconocidos por los spotters o fisonomistas ingleses deplazados a Toulouse" ( $L V, 22$ de junio de 1998; cita fiel al original en la plataforma).

13. Aunque no haremos referencia a estos casos en las secciones que siguen, en Twitter hemos registrado usos similares para señalar los deseos de observar una bella vista, de ubicar a grupos o personas del espectáculo, etc.

14. Tampoco se las registra en las herramientas del Observatori de Neologia. 
sí ocurren en tuits de hablantes de español con relación a la observación de aeronaves y al automovilismo: espoter; spotteo/spoteolespoteo; spottear/spotear/espottear/espotear.

\subsection{Ejemplos de uso}

\subsubsection{Spotter en la observación de aeronaves y su registro fotográfico}

En el terreno aeronáutico, spotter supone una asociación estrecha entre las actividades de observación inherentes a la voz inglesa y las de registro fotográfico, característica de este pasatiempo. Como hemos anticipado, denomina a la 'persona que se dedica a la identificación y posterior registro fotográfico de los distintos modelos de avión que integran las flotas de las aerolíneas (en especial, en movimiento) y también de su esquema de pintura', ocupación muy extendida en distintos países. Esta actividad recreativa y, a veces, profesional determina una relación entre sus practicantes, los spotters, y las distintas organizaciones aeronáuticas. Así, la práctica del spotting, denominación que recibe esta actividad, suele llevarse a cabo en cercanías de los aeropuertos, en puntos con buena visibilidad. En algunos aeródromos se designan incluso áreas para la observación o se organizan fechas especiales para brindar a los aficionados mejores posibilidades de acceso.

Los aficionados se agrupan en comunidades que tienen importante presencia en Internet, en las que socializan sus fotografías y videos, y pueden identificarse diferentes agrupaciones que mantienen entre sí lazos más o menos estrechos a partir del reconocimiento de una identidad compartida como interesados en la aviación. Asimismo, algunas plataformas en línea dedicadas a la aviación comercial suelen recurrir a las fotografías de estos aficionados para ilustrar sus recursos.

En cuanto al empleo de spotter en el corpus especializado de prensa de tema aeronáutico, es frecuente encontrar esta voz acompañada de una explicación, en algunos casos destacada mediante comillas o tipográficamente. Ambos fenómenos señalan el reconocimiento tanto de su carácter foráneo como de su significado no necesariamente familiar para un lector ajeno a la actividad, aspectos que sugieren un bajo grado de integración social de esta transferencia léxica. Algunos ejemplos de España y Argentina son los siguientes:

Los spotters, aficionados a observar y capturar imágenes de aviones, también podrán visitar zonas del aeropuerto a las que normalmente no se tiene acceso para conseguir una mejor perspectiva de las aeronaves. Éstos [sic] irán en todo momento acompañados y guiados por personal del aeropuerto. (La Vanguardia, 22 de junio de 2014, s/p) $)^{15}$

Ayer se difundió un video en el canal de YouTube de los spotters (quienes registran el movimiento de aviones), en el que se observa cómo un avión de la empresa rusa UTair (Boeing 767-300) estaba a punto de tocar tierra en el [sic] misma pista donde hacía su entrada un avión de Aerolíneas Argentinas (Airbus 340-300). (La Nación, 7 de julio de 2014, s/p)

15. El destacado en itálicas en estos fragmentos nos pertenece a menos que se indique lo contrario. Las negritas corresponden a los originales y los subrayados a los hipervínculos provistos por los medios de comunicación. 
La afición por fotografiar aviones es casi tan antigua como la propia aviación aunque se ha popularizado todavía más a raíz de la llegada al mercado de la fotografía digital. En El Prat, cuna de la aviación catalana, la aviación está muy extendida y funciona la primera asociación de spotters, que se reúnen cada jueves en el mirador de la playa para retratar de aviones y que suma medio centenar de socios. [...] || "Un spotter no solo fotografía el avión sino que lo identificamos, sabemos la ruta que hace, la compañia y sus características”, comenta Fernando Carrasco. (Gallego en La Vanguardia, 29 de septiembre de 2014, s/p; negritas en el original)

«Spotters»: «cazadores» de aviones o el arte magistral de fotografiarlos. (Quimbiulco en $A B C, 30 \mathrm{de}$ junio de 2015, titular)

Además de su empleo como etiqueta o hashtag (\#spotter) y como elemento formante de varios nombres de usuarios, spotter aparece con frecuencia en los textos breves que caracterizan a la red social Twitter. Así, los tuits permiten observar el empleo de esta transferencia en construcciones simples como "(no) ser + spotter" o "ir de spotter a + [aeropuerto]", así como en construcciones nominales en español o en inglés como "mundo spotter" o "Spotter Day". Como variante más adaptada al español encontramos ocurrencias de la grafía espoter, pero no de las otras formas hipotéticas para designar a persona.

\subsubsection{Spotting en la observación y fotografía de aeronaves}

La transferencia spotter se asocia con el empleo de otras unidades léxicas, algunas tomadas en su forma original del inglés y otras construidas con formantes del español a partir del lexema foráneo. Entre las primeras, hay que mencionar a la forma spotting, registrada también en el corpus de prensa aeronáutica, y que en Twitter a veces se asocia con el sustantivo plane ('avión') en la construcción plane spotting. Así, el ya mencionado sintagma "Spotter Day" coexiste en los tuits en español con la denominación de origen inglés "spotting day", para señalar una 'fecha en la que los aeropuertos invitan y autorizan a un grupo de aficionados a sacar fotografías en sus instalaciones'.

También se constata esta transferencia en la expresión "ir de spotting" o "hacer + spotting", esta última detectada también en un texto de prensa aeronáutica:

El presidente de la Asociación Spotters Barcelona-ElPrat, Fernando Olmeda, ha explicado a Europa Press que su entidad "organiza salidas durante el año para ver otros aeropuertos; luego hay 'spotters' que viajan por todo el mundo mirando de cazar el mayor número de aviones distintos y raros que puedan; y quien más quien menos aprovecha las vacaciones para acercarse a algún aeropuerto a hacer 'spotting'. (La Vanguardia, 27 de junio de 2013)

Aunque Twitter registra muchos casos de spotting como marca temática, es decir, como etiqueta (\#spotting), también es frecuente en sintagmas nominales como "punto de spotting", para referirse al 'lugar donde se puede realizar la actividad', o como "grupo de spotting" o "mundo del spotting”, que remiten al carácter social de este pasatiempo. También son habituales las 
expresiones como "sesión/día/jornada/mañana/tarde de spotting" que, como spotting day, sitúan temporalmente la actividad.

En lo que hace a su adecuación morfogramatical, spotting asume siempre género masculino en nuestras fuentes, y ocasionalmente se puede registrar la escritura de una forma de plural spottings. Por último, se ha registrado ocasionalmente la grafía spoting ("Spoting day"). ${ }^{16}$

\subsubsection{Otras expresiones: spotteo, spoteo, espoteo; spottear, spotear, espottear, espotear}

Una alternativa ocasional a spotting en Twitter es la forma spotteo, como forma nominal de género masculino que aparece en construcciones como "pronombre posesivo + spotteo" (para referirse a la práctica realizada por una persona particular), "sustantivo + de spotteo" o también "spotteo de + designación de pista de aeropuerto", entre otras que dan cuenta de las diversas posibilidades de su empleo.

También se detecta el uso de las variantes spoteo, en ocasiones en el mismo hablante que usa spotteo, y espoteo. Aparecen en sintagmas de similar naturaleza, como "sesión de + spoteolespoteo", o como etiquetas y, en ambos casos, hay adaptación a las pautas morfofonológicas y gramaticales del español para la creación de nominalizaciones derivadas de verbos de primera conjugación, a la que se incorporan las formas verbales creadas a partir de elementos léxicos ajenos al español, en este caso spottear. En espoteo, además, se representa el característico apoyo vocálico inicial en /e-/ para las transferencias léxicas con grupo inicial /sp-/.

De género masculino, spotteo y las formas relacionadas con esta expresión se pueden asociar, como acabamos de señalar, con el verbo spottear, creación léxica con adaptación a las pautas morfológicas del paradigma verbal español aunque conserva la tipografía del inglés en algunos casos. Este infinitivo ${ }^{17}$ aparece en construcciones verbales como "ir a spottear" y en complementos que califican los mejores espacios para realizar la actividad ("punto/lugar para spottear"), construcciones cuya frecuencia puede explicarse porque las tomas de aviones son difíciles de obtener si no se tiene acceso a un lugar autorizado o equipo para hacerlo desde una distancia segura en aquellas instalaciones que permiten la actividad. Otro ejemplo de interés es el aplicado a la descripción de la "cámara para spottear", que utilizan los hablantes al hacer mención del equipo fotográfico utilizado para lograr las mejores tomas fotográficas en la actividad. A nivel discursivo, esta construcción enfatiza que la actividad no se agota en la observación sino que resulta distintivo y central el registro visual de los avistajes.

Según se observó en el párrafo precedente, se ha identificado también el uso de spotear, y también de espottear y espotear en sintagmas similares: "día para spotear", "ir a spotear", "ganas de espottear". Salvo en dos ocasiones, en las que espottear y espotear se registraron como formas

16. Las variantes gráficas hipotetizadas para spotting aparecen en Twitter, hasta donde pudimos observar, como errores de tipeo para Sporting -como parte de nombre de un club deportivo- o como nombre de empresa vinculada al terreno publicitario, en el que también tiene un significado específico el sustantivo inglés spot.

17. No hemos trabajado con formas conjugadas de este verbo, pero en relación con la observación de aeronaves la hemos detectado conjugada, por ejemplo, para las tres personas del singular (yo, tú, él, pero no para vos). En el sentido general de observación la hemos encontrado en más personas. 
entrecomilladas, no detectamos otros casos en los que se destacara de alguna manera alguna de estas formas -aunque Twitter no permite destacar texto de manera simple-.

Ahora bien, como se anticipó en la sección 3.2.1, en algunas variedades de español la forma spotear parece ser frecuente entre hablantes de español en contextos cotidianos no vinculados con las actividades objeto de este trabajo. En esos casos, asume el sentido de 'observar' (con frecuencia a personas, pero también, por ejemplo, un atardecer), 'ubicar/detectar' (por ejemplo, una cosa o a una persona/grupo de personas en un conjunto). Estos usos parecen ser más frecuentes en el español de México, aunque no se pudo constatar en todos los casos el origen mexicano de los usuarios en cuyos tuits detectamos este verbo fuera del contexto de la observación de aeronaves. La observación, sin embargo, es consistente con los (muy escasos) datos de uso de espotear en corpus digitales (cfr. sección 3.2.1).

\subsubsection{El spotter en el automovilismo deportivo}

Además de encontrar algunas de las formas léxicas mencionadas en el apartado precedente con similar significado en contextos comunicativos referidos al automovilismo deportivo (spotear, espottear, espotear), es también muy habitual que se registre la voz spotter. En esta última sección de análisis nos referiremos a ella en particular para precisar su significado específico en este contexto social y para mostrar algunos de sus usos posibles.

Parece razonable considerar otra de las acepciones de spotter en inglés para explicar su empleo en este ámbito del deporte: según el Webster's, esta voz designa también a 'la persona que asiste para evitar el daño de un gimnasta'. De manera similar, en el deporte motor, puede proponerse que un spotter es 'la persona que ayuda a un piloto de autos de carreras a valorar la conveniencia estratégica de una maniobra, por lo general de sobrepaso, en función del tráfico que lo rodea o que le advierte de las incidencias ocurridas más adelante de su posición, para evitar una colisión'. En ambos casos, el spotter asiste al piloto y lo protege. Puede argumentarse, también, que el empleo de esta voz en este terreno deportivo nace también de un vínculo metafórico con la observación militar, puesto que la función del spotter es central para la estrategia de una escudería frente a la de sus adversarios.

En relación con este ámbito de uso, Twitter permite registrar sintagmas como "ser + spotter" para señalar el rol de una persona dentro del deporte, pero consideramos que resultan especialmente relevantes construcciones como "hacer de/trabajar como + spotter", puesto que, por lo general, aparecen en el discurso en contextos que sugieren que esta función la asume una persona con experiencia o que posee una relación de confianza con el piloto o el equipo. Así, la función de spotter en este deporte se asocia con acciones habitualmente expresadas por verbos de habla como decir, informar y aconsejar, directamente vinculados con el rol que cumple esta persona en el deporte y, en el caso de aconsejar, el verbo señala la principal función comunicativa asociada a este rol en el automovilismo.

Por otra parte, encontramos también sintagmas en los que la transferencia léxica cumple la función de objeto como "tener + spotter", que suele emplearse para referir a la relación entre el piloto 
y su asistente de observación como característica distintiva de algunas categorías automovilísticas, en particular de las series Indy y Nascar de Estados Unidos. Asimismo, algunos tuits que pudimos detectar señalan la importancia de esta función mediante una construcción privativa "sin + spotter", que aparece en tuits que destacan que los pilotos de otras categorías automovilísticas tienen la desventaja de no contar con la asistencia o ayuda de este tipo de colaboradores.

En un sentido similar, la importancia del aporte de estos asistentes se ve reflejada en sintagmas en los que se alude al "audio/reacción + de $(1)+$ spotter", claves para que un piloto realice la mejor maniobra. En lo que hace a la adaptación morfogramatical de esta transferencia y a su representación escrita, un caso de particular interés que tenemos constatado es el complemento "de los \#spotter's", con un plural irregular en inglés que denota el origen extranjero de la expresión.

Entre las formas que reflejan diverso grado de adaptación al español en Twitter registramos tanto espoter como espotter, como ocurre también en el caso de la fotografía de aeronaves, con ejemplos de su empleo como sustantivo masculino pero también femenino. En alguna ocasión, estas formas léxicas aparecen entre comillas y en un tuit especialmente interesante encontramos, además, un comentario metalingüístico con el que un hablante se pregunta por la correcta realización oral de "espoter".

\section{Conclusiones}

Según lo revela la inclusión de definiciones o aclaraciones junto a la voz spotter en la prensa especializada en aviación -un eslabón importante para el ingreso tanto de las expresiones foráneas como de las especializadas en el léxico general de la lengua-, no puede considerarse que esta palabra sea una transferencia léxica integrada socialmente. Sin embargo, su frecuencia en la red social Twitter permite proponer que sí se trata de un préstamo léxico entre quienes se dedican a esta actividad y se identifican con esta denominación como miembros de un grupo.

Por su parte, en el terreno del automovilismo deportivo -muy similar en sus características de alto desarrollo técnico y profesionalismo a la aviación- también se detecta un empleo extendido de esta expresión, que asume una connotación diferente en tanto designa a uno de los colaboradores más importantes para los pilotos de algunas categorías del deporte. Cabe observar que nuestra indagación partió de una revisión de los usos vinculados con el transporte aéreo y del hallazgo fortuito de esta voz en relación con el deporte automovilístico, pero el camino podría haber sido inverso. En tal sentido, resta precisar, en futuros trabajos, la relación de precedencia temporal en el uso de spotter en ambos contextos y, de manera más general, en otros deportes que aparecen mencionados en algunos tuits que incluyen esta expresión y que, por los alcances planteados para este artículo, no fueron considerados. Entre ellos se cuentan el levantamiento de pesas, los ejercicios gimnásticos en la práctica de porristas, para mencionar dos de ellos en los que se aplica con claridad la acepción deportiva que la voz tiene en inglés. Para finalizar la consideración de los contextos de uso de spotter, podemos señalar, por último, que también contamos con indicios de su empleo en relación con el avistaje de aves. Sin embargo, desconocemos todavía si, en este caso se trata de un empleo extendido entre los aficionados a esta actividad o no. Para ello serán también necesarias nuevas exploraciones en Twitter 
y en otro tipo de textos, incluyendo los periodísticos, a fin de relevar las ocurrencias de spotter asociadas con este ámbito.

Como conclusión de tipo metodológico, consideramos importante destacar que la proposición de formas léxicas hipotéticas relacionadas de spotter, con distintos grados de adaptación a las pautas morfofonológicas y ortográficas del español resultó productiva puesto que, si bien fueron escasas las formas constatadas en corpus digitales, pudimos, en cambio, identificarlas en producciones de hablantes de español en la red social Twitter. Su ocasional marcación a través del entrecomillado permite señalar que estas expresiones se perciben como creaciones léxicas recientes, incluso entre quienes se dedican a las actividades objeto de análisis en este trabajo.

En lo que hace a la posibilidad de su empleo en contextos diferentes a los considerados, fuera de Twitter, hemos constatado el empleo del verbo spotear en otro contexto deportivo que merece ser mencionado porque en él se presenta un significado algo diferente a los analizados en este trabajo y se propone, asimismo, una explicación parcialmente diferente sobre su origen. Se trata de la escalada deportiva, en la que lo que se observa no es el desempeño de un deportista (por su seguridad o en su carácter de rivales deportivos como en el automovilismo deportivo). Por el contrario, en la escalada lo que se observa es el medio en el que se realiza la actividad. Así, un hablante de 44 años de edad que se dedica a este deporte en Argentina, nos ha explicado el significado que tiene el verbo spotear en el marco de esta actividad con las siguientes palabras:

Habilidad de detectar visualmente o de "ver inmediatamente" determinados lugares, formas y aspectos de la roca y de todo el muro. Intuir las vías posibles. Viene de la palabra "spot" que en inglés es lugar, punto o mancha y de eso se trata spotear de ver rápido todos los spots. (Mensajería personal, 25 de marzo de 2021)

Para finalizar, proponemos que la existencia de una actividad recreativa como la que realizan los spotters -que en algunos casos puede convertirse en una actividad profesional- da cuenta de un modo particular de apropiación del transporte aéreo establecido desde un lugar de interés y afición que resulta favorecido por el desarrollo y la accesibilidad a las herramientas de la fotografía digital.

\section{Referencias bibliográficas}

de- Matteis, L. M. A. (2014) «Señoritas en busca de nombre»: jerarquización de una profesión a través del léxico. Revista de Lexicografía, XX, 77-106. https://bit.ly/3octpbg.

de- Matteis, L. M. A. (2016). Representaciones sociales y discurso publicitario en torno al vuelo y al transporte aéreo. Tonos digital, 31. https://bit.ly/3kkvLUB.

de- Matteis, L. M. A. (2017). El léxico español del vuelo: un proyecto lexicográfico para el dominio del transporte aéreo. Revista de Lenguas para Fines Específicos, 23 (2), 290-312. 
de- Matteis, L. M. A. (2018). Livery, low cost y overbooking: consideraciones sobre su empleo entre hablantes de español. Artifara, 18, 65-79. https://bit.ly/3wEZWuJ.

de- Matteis, L. M. A. (2019a). Volación en las primeras décadas del siglo XX: su empleo en la prensa y en el Manual Práctico de Volación de Zuloaga. Revista Digital Internacional de Lexicología, Lexicografía y Terminologia, 2, 130-150. https://bit.ly/3wpAqtf.

de- Matteis, L. M. A. (2019b). Galicismos aeronáuticos en el español bonaerense: decolar y decolaje. Ponencia en el Primer Congreso Nacional del Español Argentino. San Carlos de Bariloche, Universidad Nacional de Río Negro, 14 al 16 de noviembre.

Fontanella de Weinberg, Ma. B. (1992). El español de América. Madrid, Mapfre.

Fresán, R. (2009). El fondo del cielo. Barcelona, Random House Mondadori. [vía CoRPES XXI]

Gallego, M. C. (29 de septiembre de 2014). E1 Prat se pone de moda entre los ‘spotters' europeos. La Vanguardia. https://bit.ly/3Cd8rhr.

Gómez de Enterría Sánchez, J. (2009). El español lengua de especialidad: enseñanza y aprendizaje. Madrid, Arco/Libros.

Hipperdinger, Y. (2001). Integración y adaptación de transferencias léxicas. Contribución al estudio del contacto lingüistico en español bonaerense. Bahía Blanca, EdiUNS.

Instituto Universitario de Lingüística Aplicada. Observatori de Neologia. Barcelona, Universitat Pompeu Fabra. https://bit.ly/2YvgRDg.

La Nación (7 de julio de 2014). Aerolíneas Argentinas asegura que "no hubo una situación de riesgo" en el cruce con el avión ruso. https://bit.ly/30rBWP1.

La Vanguardia (22 de junio de 2014). El aeropuerto de Ibiza celebra este domingo la IV jornada de spotters locales. https://bit.ly/3kDhySV.

La Vanguardia (27 de junio de 2013). El aeropuerto de E1 Prat abre las puertas a los aficionados a la fotografía aeronáutica. https://bit.ly/3otGux6.

Moreno Fernández, F. y Moreno Sandoval, A. (2017). Búsqueda de anglicismos en español estadounidense a través de Twitter. Madrid/Cambridge, Instituto de Ingeniería del Conocimiento/Observatorio de la lengua española y las culturas hispánicas en Estados Unidos.

Quimbiulco, C. (30 de junio de 2015). «Spotters»: «cazadores» de aviones o el arte magistral de fotografiarlos. $A B C$. https://bit.ly/3CfM1vZ.

Real Academia Española (2014). Diccionario de la Lengua Española, Madrid, Planeta. 23a ed. Consultado: 18 de mayo de 2018. http://dle.rae.es.

Real Academia Española. Corpus de referencia del español actual (CREA). Consultado: 18 de mayo de 2018. http://www.rae.es.

Real Academia Española. Corpus del Español del Siglo XXI (CORPES). Consultado: 18 de mayo de 2018. http://www.rae.es. 
Seco, M. (2000-2001). La importación léxica y la unidad del idioma: anglicismos en Chile y en España. Boletín de Filología, XXXVIII, 253-280. [vía CORPES XXI]

Seco, M., Andrés, O. y Ramos, G. (1999). Diccionario del español actual. Madrid, Aguilar.

Webster's Encyclopedic Unabridged Dictionary of the English Language (1996). New York, Random House.

* Lorena M. A. de- Matteis es Licenciada y Doctora en Letras por la Universidad Nacional del Sur. Investigadora Adjunta del Consejo Nacional de Investigaciones Científicas y Técnicas (CONICET) y Profesora Adjunta de "Historia de la Lengua Española" en la UNS. Sus principales líneas de investigación comprenden el estudio de la comunicación institucional en ámbito aeronáutico, las representaciones sociales en torno al transporte aéreo y el léxico español del vuelo. Desarrolló funciones docentes en "Taller de Comprensión y Producción de Discursos" y actualmente cumple tareas de extensión docente en "Metodología de la Investigación Lingüística y Literaria”, y tengo a su cargo un seminario de orientación en Lingüística para la carrera de la Licenciatura en Letras de la UNS. Desarrolló también tareas docentes de posgrado en la Universidad Nacional de Tierra del Fuego, en la Universidad Nacional del Comahue y en la Universidad Católica Argentina. Ha realizado estancias de investigación en la Universidad de Granada e intercambios docentes en la Universidad Federal Fluminense y la Universidad de Lyon 2. Dirije actualmente el Proyecto de Grupos de Investigación "Competencias comunicativas profesionales y educación superior: representaciones sociales y diagnóstico de necesidades en carreras de grado de la Universidad Nacional del Sur”, con financiamiento de la Secretaría General de Ciencia y Tecnología de la UNS (24/I284).

RECIBIDO: $11 / 05 / 2020$

ACEPTADO: 03/11/2021 